\title{
Sero-survey on Long-term Care Facility Residents Reveals Increased Risk of Sub-optimal Antibody Response to BNT162B2: Implications for Breakthrough Prevention
}

\section{Barbara Caimi}

Azienda Servizi alla Persona, Istituti Milanesi Martinitt e Stelline e Pio Albergo Trivulzio

Marco Franzetti

Legnano General Hospital, ASST Ovest Milanese

Rossella Velleca

Azienda Servizi alla Persona, Istituti Milanesi Martinitt e Stelline e Pio Albergo Trivulzio

Alessia Lai ( $\nabla$ alessia.lai@unimi.it )

University of Milan

Antonella Gatti

Azienda Servizi alla Persona, Istituti Milanesi Martinitt e Stelline e Pio Albergo Trivulzio

Rossi Pier Luigi

Azienda Servizi alla Persona, Istituti Milanesi Martinitt e Stelline e Pio Albergo Trivulzio

Marco D'Orso

University of Milan-Bicocca

\section{Fabrizio Pregliasco}

University of Milan and IRCCS Istituto Ortopedico Galeazzi

Claudia Balotta

University of Milan

Giuseppe Calicchio

Azienda Servizi alla Persona, Istituti Milanesi Martinitt e Stelline e Pio Albergo Trivulzio

\section{Research Article}

Keywords: SARS-CoV-2, Long Term Care Facilities, aging, comorbidities, S-IgG antibodies

Posted Date: September 3rd, 2021

DOl: https://doi.org/10.21203/rs.3.rs-775688/v1

License: (c) (i) This work is licensed under a Creative Commons Attribution 4.0 International License. 
Version of Record: A version of this preprint was published at BMC Geriatrics on March 10th, 2022. See the published version at https://doi.org/10.1186/s12877-022-02884-0. 
1 Sero-survey on long-term care facility residents reveals increased risk of sub-optimal antibody response to

2 BNT162B2: implications for breakthrough prevention

3 Barbara Caimi ${ }^{1 *}$, Marco Franzetti ${ }^{2 *}$, Rossella Velleca ${ }^{1 *}$, Alessia Lai ${ }^{3 *}$, Antonella Gatti ${ }^{1}$, Pier Luigi Rossi ${ }^{1}$,

4 Marco D'Orso ${ }^{4}$, Fabrizio Pregliasco ${ }^{5}$, Claudia Balotta ${ }^{3}$, Calicchio Giuseppe ${ }^{1}$

51 Azienda Servizi alla Persona, Istituti Milanesi Martinitt e Stelline e Pio Albergo Trivulzio, Milan, Italy

$6 \quad 2$ Infectious Diseases Unit, Legnano General Hospital, ASST Ovest Milanese, Legnano, Italy

73 Department of Biomedical and Clinical Sciences L. Sacco, University of Milan, Milan, Italy

84 Department of Medicine and Surgery, University of Milan-Bicocca, Monza, Italy

95 Department of Biomedical Sciences, University of Milan and IRCCS Istituto Ortopedico Galeazzi, Milan, Italy

$10 *$ These authors contributed equally to this work.

11 Declaration of interest: none

12 Corresponding author: Dr. Alessia Lai

13 Department of Biomedical and Clinical Sciences L. Sacco, University of Milan, Milan, Italy

14 Via G.B. Grassi, 74

1520157 Milan

16 email: alessia.lai@unimi.it

17 Key words: SARS-CoV-2, Long Term Care Facilities, aging, comorbidities, S-IgG antibodies. 
Background: The impact of coronavirus disease 2019 caused by Severe Acute Respiratory Syndrome

Coronavirus 2 on hosts of Long Term Care Facilities (LTCFs) has been dramatic at global scale as aging and comorbitities pose individuals at increased risk of severe disease and death.

Methods: Aim of this study was to evaluate SARS-CoV-2 S-IgG antibodies titers in 478 residents and 649 health care workers of the largest Italian nurse facility two months after the complete vaccination with BNT162B2. Associations among host-related factors and predictors of humoral response were investigated. Results: By stratifying levels of humoral responses, we found that $62.1 \%, 21.6 \%, 12.1 \%$ and $4.2 \%$ of hosts has high (>1,000 BAU/ml), medium (101-1,000), low (1-100) and null (<1 BAU/mL) S-IgG titers, respectively. Hosts with previous COVID-19 and those with SARS-CoV-2 N-IgG positive serology showed higher level of serological response $(p<0.001$ and $p<0.001$, respectively), while the administration of corticosteroid or cancer diminished all levels of specific antibodies ( $p=0.019$ and $p=0.004)$. Significant associations were observed for these parameters in those with suboptimal response $(p<0.001, p<0.001, p=0.028$ and $p=0.005)$ and with a null one $(p=0.005, p<0.001$ and $p=0.039)$. Predictors of an increased risk of null response were advanced age, corticosteroid therapy and diabetes mellitus ( $p=0.025, p=0.017$ and $p=0.037)$. In contrast, previous diagnosis of COVID-19 resulted strongly associated with a reduced risk of null response to vaccination $(p<0.001)$. Conclusions: SARS-CoV-2 specific antibodies in elderly individuals need to be measured to consider a third dose of vaccine after mass vaccination for prevention of reinfections in LTCFs despite the maintenance of barrier measures.

\section{INTRODUCTION}

The coronavirus disease 2019 (COVID-19) pandemic, which started in late 2019 in China, continues to spread worldwide, despite the adoption of personal protective equipment (PPE), subsequent lockdowns, prolonged control measures implemented in most developed countries and unprecedented vaccination campaigns. The impact of COVID-19 on older people living in nursing Long Term Care Facilities (LTCFs) has been particularly 
51 as PPE for hosts and health care workers (HCWs) and the health vulnerabilities of the elderly, all contributed

52 to the spread and lethality of the virus in the setting of Nurse Facilities (NFs). In addition, conditions like

53 frailty, dependence, dementia or high burden of comorbidities are responsible of high incidence of disease

54 susceptibility and mortality. By July 2021, the highest mortality rate among older people (aged $>75$ years), has reached $48.7 \%$ of confirmed cases as reported by New York City Health (Worldometer. Age, sex, existing conditions of COVID-19 cases and deaths (https://www.worldometers.info/coronavirus/coronavirus-agesex-demographics/ (accessed July 8, 2021). In parallel, Italian data from the Istituto Superiore di Sanità (from February 2020 to May 2021) indicate that the overall mortality rate, defined as number of deaths over the total of residents, was 9.1\% (https://www.epicentro.iss.it) coronavirus > pdf).

Age, concomitant pathologies and immunosenescence are recognized as the main factors that influence the cellular immune responses and alteration of lymph node architecture play a major role in the failure to contrast pathogens and related morbidity [2]. Indeed, immune responses may be affected both by aforementioned factors and other common events related to senescence, such as the alteration of metabolic processes, blood circulation, gas exchanges and organ function. Lower responses to influenza virus, Streptococcus pneumoniae and some Flaviviruses have been demonstrate in elderly compared to younger adults [3-8]. For these reasons elderly and hosts of nursing facility, as well as HCWs taking care of them, have been prioritized to be vaccinated as soon as vaccines became available (December 27, 2020). Several reports indicate that vaccination of old age is showing to protect from severe disease, and risk of

71 death by conferring a degree of humoral immunity [9-15]. below 60 years by investigating differences in antibody titers by age, and we identify factors related to 
important to reinforce protective measures in $\mathrm{NHs}$ as well as to pose the need of to identify effective

77 vaccination strategies in frail residents, including additional doses and/or switch to different vaccines.

\section{METHODS}

79

80

81

82

\section{Study population}

The study was conducted at the Pio Albergo Trivulzio, the main LTCF in Milan, hosting about 500 aged residents and hiring about $700 \mathrm{HCW}$. Four hundred and seventy-eight LTCF residents and $649 \mathrm{HCW}$ sere studied. HCWs included nurses, doctors, healthcare technicians, health service assistant, cleaners, laboratory and administrative staff.

First dose administration of BNT162b2 vaccine started in the structure on December, 27 and occurred until January, 31. Second dose was administered at 21 days and was completed on February 25.

Around two months after the second dose, a blood sample was obtained for routine assessment of laboratory parameters and a $500 \mu \mathrm{l}$ were stored at $-20^{\circ}$ for SARS-CoV-2 antibody quantifications. HCWs were vaccine administered and tested in parallel according to an informed consent. Samples were concomitantly screened for SARS-Cov-2 nucleocapsid protein (N-IgG) to capture possible asymptomatic infection or assess the persistence of specific antibodies in previously infected subjects. All data used in this study were previously anonymized as required by the Italian Data Protection Code (Legislative Decree196/2003) and the general authorizations issued by the Data Protection Authority. Ethics Committee approval was deemed unnecessary because, under Italian law, it is only required in the case of prospective clinical trials of medical products for clinical use (Art. 6 and Art. 9 of Legislative Decree 211/2003). All patients gave their written informed consent to the medical procedures/interventions carried out for routine treatment purposes. The study was conducted in compliance with Good Clinical Practice and the declaration of Helsinky.

\section{SARS-CoV-2 antibody assays}

We used Elecsys Anti-SARS-CoV-2 S (Roche), an immunoassay for the quantitative determination in vitro of total antibodies to the SARS-CoV-2 spike (S) protein receptor binding domain (RBD) in human serum and 
101 plasma. The assay cut off is $>0.8 \mathrm{BAU} / \mathrm{ml}$ reported by manufacturer. Elecsys Anti-SARS-CoV-2 S assay 102 manufacturer reports a sensitivity of $98.8 \%$ (95\% Cl: $98.1-99.3 \%$ ) and a specificity of $95 \%$ (Cl: 99.7 $103100 \%)$.

104 Response to vaccination in residents was classified as high, medium, low and null response by stratifying the 105 level of anti-S IgG values in 4 levels: $>1,000,101-1,000,1-100$ and $<1 \mathrm{BAU} / \mathrm{mL}$, respectively.

106 Elecsys Anti-SARS-CoV-2 (Roche), an immunoassay for the in vitro quantitative determination of antibodies 107 to the SARS-CoV-2 N protein in human serum and plasma was used with a sensitivity of 99.5\% (Cl: $97.0-100 \%)$ 108 and a specificity of $99.80 \%(\mathrm{Cl}: 99.69-99.88)$.

\section{Statistical analysis}

110 Parametric tests ( $t$ test and ANOVA), nonparametric tests (Mann-Whitney and Kruskal-Wallis) and the 111 Pearson $\chi 2$ test (or Fisher exact test, when necessary) were used to compare normally distributed, non112 normally distributed continuous, and categorical variables of patients, respectively. The primary endpoint 113 was the risk of null response to vaccine, evaluated by means of a logistic regression model, also correcting 114 for gender, age, comorbidities and immune modulatory treatments. Also, previous diagnosis of COVID-19 115 and positive anti-nucleocapsid serology were included as correlates in the analysis in two different models, 116 due to their strong reciprocal association. We evaluated their combined effect over the risk of null response 117 to vaccination stratifying the population based on previous clinical and serological evidence of SARS-CoV-2 118 exposure.

\section{RESULTS}

\section{Participant characteristics}

\section{Hosts}

122 The main demographic and clinical characteristics of 478 analyzed LTCF subjects are shown in Table 1 . The 123 majority of residents were females ( $81 \%)$ with an age higher than 80 years old ( $n=345,72.2 \%)$. SARS-COV-2 124 anti-nucleocapsid antibodies determination was available for 455 subjects and resulted positive in 268 cases 
125 (58.9\%). Among these, 143 hosts (53.4\%) did not show clinical signs of infection in the past, while 7 patients 126 with documented clinical COVID-19 resulted negative for serology $(7 / 187,3.7 \%)$.

127 A large majority $(n=413,86.4 \%)$ of residents presented at least one morbidity, being affected by dementia, 128 the most common syndrome, in $58.6 \%$ of cases. Almost all hosts received polytherapies for chronic diseases 129 (hypertension, heart disease, lung disease, diabetes and cancer). Anticoagulant or corticosteroid therapy 130 resulted administered in $114 / 478$ (23.8\%) and 19/478 (4.0\%), respectively.

131 HCWs

132 Among the $649 \mathrm{HCWs}$, the majority of subjects were females ( $n=464,71.5 \%)$ with a median age of 49 years 133 (IQR: $34-55)$. In detail, the age classes were as follow: 15.1\% ( $n=98), 18.2 \%(n=118), 21.6 \%(n=140), 36.8 \%$ $134(n=239)$ and $8.3 \%(n=54)$ for $\geq 30,31-40,41-50,51-60$ and $>60$ years, respectively. About one third of subjects $135(27.3 \%, \mathrm{n}=113)$ presented at least one comorbidity, while 14 presented two of them. HCWs with a previous exposure to SARS-CoV-2 were 124 (19.1\%) as detected by nasopharyngeal swab (data not shown).

\section{Vaccine-associated side effects}

138 No major vaccine associated side effects occurred either in residents or in HCWs. Minor effects such as site 139 of injection pain, fatigue, malaise, headache, nausea or skin rash, muscle and joint pain, fever were reported 140 in about $10 \%$ and $16 \%$ of individuals, respectively.

141 Serological response to vaccine

142 Hosts

143 Overall, we detected anti-S-IgG antibodies in $95.8 \%$ of hosts receiving two doses of BNT162b2 vaccine. 144 According to level of response, high, medium and low titers were present in $62.1 \%(n=297), 21.6 \%(n=103)$ 145 and $12.1 \%(n=58)$ of cases, respectively.

146 Median level of anti-S IgG of 7.500, corresponding to the upper limit of quantification of the assay (IQR: 147 848- >7.500 BAU/mL) after a median time of 64 days from the second dose (IQR 63-65 days).

148 We first investigated the associations among main epidemiological and clinical characteristics and levels of 149 response to vaccination. The results are reported in Table 2. 

and diabetes $(10.4 \%$ vs. $3 \% p=0.052)$. A significantly higher response was detected for host with previous COVID-19 and for those with SARS-CoV-2 N-IgG serology considering all level of serological response $(p<0.001$ and $p<0.001)$. In contrast, the administration of corticosteroid diminished all levels of specific antibodies $(p=0.019)$. Significant associations were observed for these parameters in those with suboptimal response $(p<0.001, p<0.001$ and $p=0.028)$ and with a null one $(p=0.005, p<0,001$ and $p=0.039)$.

Among subjects with a previous COVID-19 clinical diagnosis, we did not find any case of null response to vaccine, either in those with positive or negative nucleocapisd serology (0/125 and 0/7, respectively). Differently, among hosts without a documented previous diagnosis of COVID-19, null response to vaccine was lower in those with positive nucleocapisd serology when compared to subjects with negative serology: $0.7 \%(1 / 143)$ vs. $10.6 \%(19 / 180)$, respectively $(p<0.001)$ (data not shown).

LTCF residents with neoplastic disease showed a significant difference in the distribution of antibody response considering all levels $(p=0.004)$ and suboptimal response $(p=0.005)$ but not null response.

\section{HCWs}

164 Regarding the response to vaccination, $66.1 \%(n=429)$ of subjects showed high titers and $33 \%(n=214)$ 165 medium titers. A suboptimal response was observed in 6 subjects (0.9\%), of whom 2 were null responder. 166 The median level of anti-S IgG in HCW was 1.789 (IQR: 754->7.500), markedly lower compared to hosts, 167 according to the lower percentage of previously infection in these subjects. A significantly different 168 distribution of response was detected for age classes both considering all titer strata $(p<0.001)$ and null 169 response $(p=0.040)$. Null responders were present only in subjects with more than 60 years $(n=2,3.7 \%)$. A 170 significantly different distribution of response was present for previous COVID-19 only considering all strata 171 of antibody titers $(\mathrm{p}<0.001)$, as the only two non-responders did not experience COVID-19 (data not shown).

\section{Predictors of responses in LTCF hosts and HCWs}

173 We then studied predictors of null response to SARS-CoV-2 vaccination by univariate and multivariate logistic 174 regression analysis. Firstly, the advanced age resulted strongly associated with an increased risk of null 
response ( $p=0.005$ and $p=0.025$ in univariate and multivariate analysis, respectively). In addition, corticosteroid therapy resulted associated with no anti-spike antibody titers in both analyses $(p=0.17$ and $p=0.042$ ). Diabetes mellitus resulted significantly associated with a higher risk of null response only in the multivariate analysis $(p=0.037)$.

Further, previous diagnosis of COVID-19 resulted strongly associated with a reduced risk of null response to vaccination $(p<0.001)$. No association was observed for cancer and female sex either in the univariate or multivariate analyses.

When evaluating positive anti-nucleocapsid serology instead of COVID-19 clinical diagnosis, the former resulted associated with a lower risk of null response to vaccine, both in the univariate $(\mathrm{OR} 0.035,95 \% \mathrm{Cl}$ : 0.01-0.26) and in the multivariate analysis (OR $0.051,95 \% \mathrm{Cl} 0.01-0.42$ ), with no impact on significance of other covariates (data not shown).

As for HCW univariate and multivariate analyses did not show any factor associated with null response to vaccination (data not shown).

\section{DISCUSSION}

Surveys are of great importance to define the serological effects to COVID19 vaccine in real world population, particularly in fragile individuals. In general, quantification of the antibody response to SARS-CoV-2 vaccination is highly relevant for identifying possible vaccine failure (i.e the risk of breakthrough infection) and estimating level and time of protection. However, thresholds for positivity and cut off values provided by different assay manufacturers differ and their diagnostic value is not yet established and standardized at present. Although total anti-spike titers may be not indicative of sufficient inhibitory capacities, vaccinationinduced antibody titers may be used as surrogate marker from which a protection correlate could be estimated $[16,17]$.

Several papers addressed the efficacy of different SARS-CoV-2 vaccines either through Phase II and III of clinical trials [18-20] or field investigations [9-11]. Few of them detailed data regarding elderly and persons living in nurse facility [12-15] and outbreaks after the first and second dose were reported [21-23]. At 
present, cohorts $<16$ years or $>80$ years who might show reduced vaccine reactiveness are limited. We report

201 a large sero-survey in residents of the largest LTCF of Italy where SARS-CoV-2 could spread because of late warning and lack of PPE and diagnostic tools. Our data indicate that full vaccination either in LTCF hosts and HCWs elicits a humoral response in above $96 \%$ of individuals accordingly with published papers. Nevertheless, while the majority of both elderly vaccinees and HCWs raised high responses after their second vaccination dose, a highest percentage of hosts showed a lower or null response when compared to HCW (14.2\% vs. $0.9 \%)$. The main differences between the two groups of vaccinated individuals are likely a consequence of immunosenescence, which describes the phenomenon of reduced adaptive immune responses in hosts. Previous data reported that titers of S-IgG antibodies are significantly lower in elderly persons [18]. Accordingly, our regression analysis in LTCF residents indicates that aging is strongly associated with an increased risk of null response.

211 Noteworthy, our findings suggest that comorbidities and their treatment may impact humoral responses as 212 detected by several significant associations, even though only diabetes and corticosteroid therapy confer an 213 increased risk of unresponsiveness. Limited numbers of LTCF hosts with other morbidities such cancer and 214 diseases of the immune system prevent us to detect other associations impacting antibody response. To our 215 knowledge no similar data are yet available in elderly persons.

216 Our study population includes older adults living in assisted structures and their care providers, with or 217 without a history of natural infection with SARS-CoV-2. As already reported, individuals having a history of 218 natural infection or positive serology have higher antibody levels and an enhanced response to vaccination, 219 even after a single dose [24]. Indeed, in our case-file both prevalence of diagnosed COVID-19 and median titers of S-IgG were higher in hosts compared to HCWs $(56 \%$ vs. $19.1 \%$ and 7.500 vs. $1.789 \mathrm{BAU} / \mathrm{mL}$, 221 respectively). Consequently, both N-IgG positive results and previous COVID-19 resulted predictive factors of 222 positive response to vaccination.

223 Although serological assays may still need to be standardized, compared and interpreted in the light of large 224 population results, they allow to preliminarily evaluate levels of protection conferred by SARS-CoV-2 
vaccines. As demonstrated for other vaccine campaigns, vaccination of old individuals often fails to induce

226 high titers of antibodies or fully protective immunity as quality and quantity of antibody titers may be 227 markedly inferior in elderly compared to adults $[2-8,13,25]$.

228 The major limitation of our study is that we measured spike (S) protein RBD antibodies, stratifying the level 229 of response in high, medium, inadequate and null instead of evaluating neutralizing antibodies that are 230 considered the better correlate of protection. Further differences in humoral response may be underscored 231 in our study as a high percentage of values are above the upper limit of the assay quantification. We could 232 not address this point by dilution experiments because of the large number of studied subjects. In addition, 233 we measured S-IgG antibodies around two months after the vaccine administration. It is conceivable that 234 antibodies titers could be above the ELISA dynamic range. Future studies to monitor antibody levels will 235 clarify the dynamic of antibody mount and decay.

236 Our considerations are based on the hypothesis anti-SARS-CoV-2 antibodies are the major correlate of 237 protection. Indeed, immunity is a complex phenomenon where both humoral and cellular responses are 238 interdependent involving both innate and adaptive immunity. Further studies will be essential to understand 239 type and function of antibodies produced after vaccination, the neutralizing capacity of the antibodies along 240 with the persistence of their protective effects. Therefore, information regarding S-IgG levels are as much 241 crucial as the identification of SARS-CoV-2 emerging variants that may elude protection conferred by 242 vaccines. Although the effectiveness of mRNA vaccines may be inferred by screening methods based on 243 sources of population-based data, we believe that accurate results from standardized assays are essential at 244 individual level to protect elderly hosts from SARS-CoV-2 reinfection [26].

245 Old subjects who have been heavily hit by the SARS-CoV-2 pandemic need to be protected through general 246 prevention actions and specific measures that include early vaccine administration and testing of their effects 247 on humoral responses. Furthermore, as demonstrated in individuals with solid organ transplantation [27], a $2483^{\text {rd }}$ dose of homologous vaccine needs to be administered in unresponsive populations.

\section{ABBREVIATIONS}


251 COVID-19: Coronavirus disease 2019

252 PPE: Protective Equipment

253 HCWs: Health Care Workers

254 NFs: Nurse Facilities

255 S: Spike protein

256 RBD: Receptor Binding Domain

257 N: Nucleocapisd protein

258 IQR: Inter-Quartile Range

259 COPD: Chronic Obstructive Pulmonary Disease

260 Cl: Confidence Interval

261 OR: Odd Ratio

262 ACKNOWLEDGEMENT

263 We thank FR Simonetti for useful discussion of manuscript.

264 AUTHORS' CONTRIBUTIONS

$265 \mathrm{BC}, \mathrm{RV}, \mathrm{AG}, \mathrm{PLR}, \mathrm{MD}, \mathrm{FP}$ and GC collected the serological and clinical data. MF performed the statistical 266 analysis. $\mathrm{AL}$ and $\mathrm{CB}$ wrote the first version of the article. All the authors contributed to the final version of 267 the manuscript.

268 FUNDING

269 No external funding was received.

270 AVAILABILITY OF DATA AND MATERIALS

271 All data generated or analysed during this study are included in this published article and its supplementary 272 information files.

273 DECLARATIONS

274 Ethics approval and consent to participate 
275 All data used in this study were previously anonymized as required by the Italian Data Protection Code 276 (Legislative Decree196/2003) and the general authorizations issued by the Data Protection Authority. Ethics 277 Committee approval was deemed unnecessary because, under Italian law, it is only required in the case of 278 prospective clinical trials of medical products for clinical use (Art. 6 and Art. 9 of Legislative Decree 211/2003). 279 All patients gave their written informed consent to the medical procedures/interventions carried out for 280 routine treatment purposes. The study was conducted in compliance with Good Clinical Practice and the 281 declaration of Helsinky.

\section{Consent for publication}

283 Not applicable.

284 Competing interests

285 The authors declare that they have no competing interests.

286 REFERENCES

287 1. Hashan MR, Smoll N, King C, et al. Epidemiology and clinical features of COVID-19 outbreaks in aged care 288 facilities: A systematic review and meta-analysis. EClinicalMedicine. 2021; 33: 100771.

289 2. Crooke SN, Ovsyannikova IG, Poland GA, Kennedy RB. Immunosenescence and human vaccine immune 290 responses. Immun Ageing. 2019; 16:25.

291 3. Goodwin K, Viboud C, Simonsen L. Antibody response to influenza vaccination in the elderly: a quantitative 292 review. Vaccine. 2006; 24:1159-69.

293 4. Schenkein JG, Park S, Nahm MH. Pneumococcal vaccination in older adults induces antibodies with low 294 opsonic capacity and reduced antibody potency. Vaccine. 2008; 26: 5521-6.

295 5. Siegrist CA, Aspinall R. B-cell responses to vaccination at the extremes of age. Nat Rev Immunol. 2009; 9 : $296 \quad 185-94$.

297 6. Sasaki S, Sullivan M, Narvaez CF, et al. Limited efficacy of inactivated influenza vaccine in elderly individuals 298 is associated with decreased production of vaccine-specific antibodies. J Clin Invest. 2011; 121: 3109-19. 
7. Stiasny K, Aberle JH, Keller M, Grubeck-Loebenstein B, Heinz FX. Age affects quantity but not quality of 300 antibody responses after vaccination with an inactivated flavivirus vaccine against tick-borne encephalitis. 301 PLoS One. 2012; 7: e34145.

8. Zimmermann P, Curtis N. Factors That Influence the Immune Response to Vaccination. Clin Microbiol Rev. 2019; 32: e00084-18.

9. Haas EJ, Angulo FJ, McLaughlin JM, et al. Impact and effectiveness of mRNA BNT162b2 vaccine against SARS-CoV-2 infections and COVID-19 cases, hospitalisations, and deaths following a nationwide vaccination campaign in Israel: an observational study using national surveillance data. Lancet. 2021; 397: 1819-1829.

10. Abu Jabal K, Ben-Amram H, Beiruti K, et al. Impact of age, ethnicity, sex and prior infection status on immunogenicity following a single dose of the BNT162b2 mRNA COVID-19 vaccine: real-world evidence from healthcare workers, Israel, December 2020 to January 2021. Euro Surveill. 2021; 26: 2100096.

11. Alpert EA, Herbst R, Abramovich I, Strugo R, Jaffe E. Mass COVID-19 vaccination of residents in geriatric 311 facilities by emergency medical services: the Israeli experience. Lancet Healthy Longev. 2021; 2: e189-e190. 312 12. Moustsen-Helms IR, Emborg H, Nielsen J, et al. Vaccine effectiveness after 1st and 2nd dose of the 313 BNT162b2 mRNA Covid-19 Vaccine in long-term care facility residents and healthcare workers - a Danish 314 cohort study. medRxiv 2021. https://www.medrxiv.org/content/10.1101/2021.03.08.21252200v1. Accessed 31502 Aug 2021.

13. Brockman MA, Mwimanzi F, Sang Y, et al. Weak humoral immune reactivity among residents of long-term 317 care facilities following one dose of the BNT162b2 mRNA COVID-19 vaccine. medRxiv [Preprint]. 2021 Mar $318 \quad 24: 2021.03 .17 .21253773$.

319 14. Salmerón Ríos S, Mas Romero M, Cortés Zamora EB, et al. Immunogenicity of the BNT162b2 vaccine in 320 frail or disabled nursing home residents: COVID-A study. J Am Geriatr Soc. 2021; 69: 1441-1447

321 15. Van Praet JT, Vandecasteele S, De Roo A, De Vriese AS, Reynders M. Humoral and cellular immunogenicity 322 of the BNT162b2 mRNA Covid-19 Vaccine in nursing home residents. Clin Infect Dis. 2021: ciab300. 
324 Antibody Levels: a Head-to-Head Comparison of Five Quantitative Assays. Microbiol Spectr 2021: e0024721.

325 17. Patel EU, Bloch EM, Clarke W, et al. Comparative performance of five commercially available serologic assays to detect antibodies to SARS-CoV-2 and identify individuals with high neutralizing titers. J Clin Microbiol. 2021;59: e02257-20.

18. Polack FP, Thomas SJ, Kitchin N, et al. Safety and efficacy of the BNT162b2 mRNA Covid-19 vaccine. N Engl J Med. 2020; 383: 2603-15.

19. Baden LR, El Sahly HM, Essink B, et al. Efficacy and safety of the mRNA-1273 SARS-CoV-2 vaccine. N Engl J Med. 2021; 384: 403-16.

20. Folegatti PM, Ewer KJ, Aley PK, et al. Safety and immunogenicity of the ChAdOx1 nCoV-19 vaccine against SARS-CoV-2: a preliminary report of a phase $1 / 2$, single-blind, randomised controlled trial. Lancet 2020; 396:467-478. Erratum in: Lancet. 2020; 396: 466. Erratum in: Lancet. 2020; 396: 1884.

21. Britton A, Jacobs Slifka KM, Edens C, et al. Effectiveness of the Pfizer-BioNTech COVID-19 Vaccine Among 336 Residents of Two Skilled Nursing Facilities Experiencing COVID-19 Outbreaks - Connecticut, December 337 2020-February 2021. MMWR Morb Mortal Wkly Rep. 2021; 70: 396-401.

338 22. Cavanaugh AM, Fortier S, Lewis P, et al. COVID-19 Outbreak Associated with a SARS-CoV-2 R.1 Lineage 339 Variant in a Skilled Nursing Facility After Vaccination Program - Kentucky, March 2021. MMWR Morb Mortal 340 Wkly Rep. 2021; 70: 639-643.

341 23. Teran RA, Walblay KA, Shane EL, et al. Postvaccination SARS-CoV-2 infections among skilled nursing 342 facility residents and staff members - Chicago, Illinois, December 2020-March 2021. Am J Transplant. 2021; $343 \quad 21: 2290-2297$.

344 24. Ebinger JE, Fert-Bober J, Printsev I, et al. Antibody responses to the BNT162b2 mRNA vaccine in individuals 345 previously infected with SARS-CoV-2. Nat Med. 2021; 27: 981-984.

346 25. Müller L, Andrée M, Moskorz W, et al. Age-dependent immune response to the Biontech/Pfizer BNT162b2 347 COVID-19 vaccination. Clin Infect Dis. 2021: ciab381. 
348 26. Mazagatos C, Monge S, Olmedo C, et al. Effectiveness of mRNA COVID-19 vaccines in preventing SARS-

349 CoV-2 infections and COVID-19 hospitalisations and deaths in elderly long-term care facility residents, Spain, 350 weeks 532020 to 13 2021. Euro Surveill. 2021; 26: 2100452.

351 27. Kamar N, Abravanel F, Marion O, Couat C, Izopet J, Del Bello A. Three Doses of an mRNA Covid-19 Vaccine 352 in Solid-Organ Transplant Recipients. N Engl J Med. 2021.

353

354

355

356

357

358

359

360

361

362

363

364

365

366

367

368

369

370 
371 Table 1. Characteristics of 478 subjects undergoing to SARS-CoV-2 vaccination in a long-term care

372 facility. Categorical variables are expressed as \% (N), continuous variables as median value (Inter-

373 quartile range).

$\mathrm{n}(\%)$

\begin{tabular}{ll}
\hline \hline Gender, female & $81.0(387)$ \\
Age, years (IQR) & $87(82-92)$ \\
Age distribution & \\
$<70$ years & $5.0(24)$ \\
$70-80$ years & $11.1(53)$ \\
$80-90$ years & $47.5(227)$ \\
$>90$ years & $36.4(174)$ \\
Previous Covid-19 & $29.7(142)$ \\
Diabetes & $15.5(74)$ \\
Cancer & $4.8(23)$ \\
Malnutrition & $15.5(74)$ \\
Heart Disease & $4.0(19)$ \\
COPD & $13.4(64)$ \\
Cerebral stroke & $15.7(75)$ \\
Dementia & $58.6(280)$ \\
Autoimmune disease & $1.0(5)$ \\
Gastrointestinal/Liver Disease & $1.3(6)$ \\
Chronic kidney disease & $1.7(8)$ \\
Anemia & $5.6(27)$ \\
Immunosuppressive therapy & $0.6(3)$ \\
Anticoagulant therapy & $23.8(114)$ \\
O2 therapy & $4.8(23)$ \\
Corticosteroid therapy & $4.0(19)$ \\
Previous positive anti-nucleocapsid serology & $58.9(268 / 455)$ \\
Anti-spike antibodies median titre (IQR) & $>7500(848-7500)$ \\
\hline &
\end{tabular}

${ }^{a}$ Chronic obstructive pulmonary disease 
Table 2. Response to SARS-CoV-2 vaccination among 478 patients living in a long-term care facility, according to the main epidemiological and clinical characteristics showing

significant associations and differences among groups. Pearson $\chi 2$ test was used to compare categorical variables of patients in the study groups.

\begin{tabular}{|c|c|c|c|c|c|c|c|c|}
\hline & & \multicolumn{4}{|c|}{ Grade of response to SARS-CoV-2 vaccine, $\%(n)$} & \multicolumn{3}{|c|}{ Comparison of response distribution, $\mathrm{p}$-value } \\
\hline & & $\begin{array}{l}\text { High response } \\
n=297\end{array}$ & $\begin{array}{l}\text { Medium response } \\
n=103\end{array}$ & $\begin{array}{l}\text { Low response } \\
n=58\end{array}$ & $\begin{array}{l}\text { Null response } \\
n=20\end{array}$ & Overall* & Suboptimal response** & Null response ${ }^{* * *}$ \\
\hline \multirow{2}{*}{ Gender } & Females & $61.7(239)$ & $22.5(87)$ & $10.9(42)$ & 4.9 (19) & \multirow{2}{*}{ ns } & \multirow{2}{*}{ ns } & \multirow{2}{*}{0.050} \\
\hline & Males & $63.7(58)$ & $17.6(16)$ & $17.6(16)$ & $1.1(1)$ & & & \\
\hline \multirow{4}{*}{ Age groups, years } & $<70$ & $58.3(14)$ & $29.2(7)$ & $8.3(2)$ & $4.2(1)$ & \multirow{4}{*}{ ns } & \multirow{4}{*}{ ns } & \multirow{4}{*}{0.061} \\
\hline & $70-80$ & $62.3(33)$ & $20.8(11)$ & $113.2(7)$ & $3.8(2)$ & & & \\
\hline & $80-90$ & $63.4(144)$ & $50.0(22)$ & $11.9(27)$ & $2.6(6)$ & & & \\
\hline & $>90$ & $60.9(106)$ & $20.1(35)$ & $12.6(22)$ & $6.3(11)$ & & & \\
\hline \multirow{2}{*}{ Previous Covid-19 } & No & $49.1(165)$ & $28.9(97)$ & $16.1(54)$ & $5.9(20)$ & \multirow{2}{*}{$<0.001$} & \multirow{2}{*}{$<0.001$} & \multirow{2}{*}{0.005} \\
\hline & Yes & $92.9(132)$ & $4.2(6)$ & $2.8(4)$ & $0.0(0)$ & & & \\
\hline \multirow{2}{*}{ Anti-Nucleocapsid serology } & Neg & $17.1(32)$ & $47.6(89)$ & $25.1(47)$ & $10.2(19)$ & \multirow{2}{*}{$<0.001$} & \multirow{2}{*}{$<0.001$} & \multirow{2}{*}{$<0.001$} \\
\hline & Pos & $98.1(263)$ & $1.1(3)$ & $0.4(1)$ & $0.4(1)$ & & & \\
\hline \multirow{2}{*}{ Diabetes } & No & $64.1(259)$ & $20.9(85)$ & $12.0(48)$ & $3.0(12)$ & \multirow{2}{*}{0.077} & \multirow{2}{*}{ ns } & \multirow{2}{*}{0.052} \\
\hline & Yes & $51.9(38)$ & $24.7(18)$ & $13.0(10)$ & $10.4(8)$ & & & \\
\hline \multirow{2}{*}{ Cancer } & No & $63.9(291)$ & $21.1(96)$ & $11.0(50)$ & $4.0(18)$ & \multirow{2}{*}{0.004} & \multirow{2}{*}{0.005} & \multirow{2}{*}{ ns } \\
\hline & Yes & $29.2(7)$ & $29.2(7)$ & $33.3(8)$ & $8.3(2)$ & & & \\
\hline \multirow{2}{*}{ Corticosteroid therapy } & No & $63.2(290)$ & $21.3(98)$ & $12.0(55)$ & $3.5(16)$ & \multirow{2}{*}{0.019} & \multirow{2}{*}{0.028} & \multirow{2}{*}{0.039} \\
\hline & Yes & $36.8(7)$ & $26.3(5)$ & $15.8(3)$ & $21.0(4)$ & & & \\
\hline
\end{tabular}

* Overall p-value: comparison between all the response groups

** Suboptimal response p-value: comparison of low-null response vs. medium-high response

*** Null response $\mathrm{p}$-value: comparison of null response vs. all the other responses grouped together 
Table 3. Logistic regression model evaluating the risk of null response to SARS-CoV-2 vaccination among

381478 patients living in a long-term care facility.

382

Age, per 10 year higher

Gender, females vs. males

Previous Covid-19

Diabetes mellitus

Cancer

Corticosteroid therapy

a Confidence interval
Univariate analysis

\begin{tabular}{llllll}
\hline \hline $\mathrm{p}$ & $\mathrm{OR}^{\mathrm{a}}$ & $95 \% \mathrm{Cl}^{\mathrm{b}}$ & $\mathrm{p}$ & $\mathrm{OR}$ & $95 \% \mathrm{Cl}$
\end{tabular}

\section{Multivariate analysis}

$\begin{array}{llllll}0.005 & 2.988 & 1.40-6.39 & 0.025 & 2.630 & 1.13-6.14\end{array}$

0.123

4.905

$0.65-37.0$

$0.292 \quad 3.103$

$0.38-25.50$

0.000

0.103

$0.01-0.12$

$0.000 \quad 0.126$

$0.02-0.23$

0.060

2.613

$0.96-7.11$

$0.037 \quad 3.415$

$1.08-10.8$

0.936

1.088

$0.14-8.53$

$0.377 \quad 0.357$

$0.04-3.51$

$0.017 \quad 3.246$

$1.23-8.54$

$0.042 \quad 4.964$

1.06-23.52 